\title{
Posterior cervical spinal fusion in a 3-week-old infant with a severe subaxial distraction injury
}

\author{
Christopher M. Holland, MD, PhD, ${ }^{1}$ Meysam A. Kebriaei, MD, ${ }^{2}$ and David M. Wrubel, MD ${ }^{1,3}$ \\ 1'Department of Neurosurgery, Emory University School of Medicine, Atlanta, Georgia; ${ }^{2}$ Children's Hospitals and Clinics of \\ Minnesota, St. Paul, Minnesota; and 'Pediatric Neurosurgical Associates, Children's Healthcare of Atlanta, Georgia
}

\begin{abstract}
Unstable spinal injuries in the neonate pose particular challenges in the clinical and radiographic assessment as well as the surgical stabilization of the spine. In this report, the authors present the unfortunate case of a 3-week-old infant who suffered a severe subaxial cervical fracture dislocation with spinal cord injury that occurred as a result of nonaccidental trauma. Imaging demonstrated severe distraction at C5-6 and near-complete spinal cord transection resulting in quadriparesis. Open surgical reduction was performed with noninstrumented posterior fusion augmented with split rib autograft and recombinant human bone morphogenetic protein-2. Postoperative imaging demonstrated progressive bony fusion at 2 months, and clinical examination findings progressed to a motor examination classification of ASIA C. At 2 years, the fusion mass is stable and cervical alignment is maintained. The patient remains flaccid in the bilateral lower extremities, but has movement with some dexterity in both hands. Follow-up MRI shows severe spinal cord injury with evidence of bilateral C-5 nerve root avulsions. This case represents the first report of spinal fusion in an infant of less than 1 month of age. Given the extreme young age of the patient, the diagnostic challenges as well as the mechanical and technical considerations of surgical fusion are discussed.
\end{abstract}

http://thejns.org/doi/abs/10.3171/2015.3.PEDS13568

KEY WORDS neonate; cervical spine injury; spinal fusion; spinal cord injury; spine

$\mathrm{Cr}_{\mathrm{r}}^{\mathrm{r}}$ ERVICAL spine injury in the pediatric population is relatively uncommon, with a reported incidence of around $1 \%$ of trauma patients. ${ }^{12,14}$ Motor vehicle accidents are the leading cause of injury in older children. However, severe spinal cord injury in neonates historically resulted as a complication of breech deliveries. ${ }^{2}$ More recently, the majority of neck injuries in very young children have resulted from falls. ${ }^{12}$ In children suffering from both accidental and nonaccidental trauma, injury most often occurs in the upper cervical spine and craniocervical junction. ${ }^{4}$

Unique anatomical differences in young children that include incomplete ossification of the spine, ligamentous elasticity, and disproportionate head weight predispose them to more severe and more often complete spinal cord injuries. ${ }^{3}$ For this reason, complete spinal cord injuries are common in the pediatric population, with several studies demonstrating a rate between $60 \%$ and $69 \%$ of pediatric patients with cervical spinal cord injuries. ${ }^{3,8,16}$
Identification of cervical injuries in very young children is difficult due to limitations of the neurological examination in this age group. There is inherent difficulty in distinguishing volitional from reflexive movements, in the detection of sensory deficits, and in the assessment of motor function in specific muscle groups. Further, radiographic studies can fail to identify cases in which severe ligamentous and spinal cord injury occur in the absence of detected bony abnormality. ${ }^{9}$

Reports of surgical intervention for cervical spine injury in the infant population are extremely rare. A review of the literature yields a single operative case report involving a child who was 4 month of age and reports of halo fixation, traction, and external bracing in which the youngest child was $21 / 2$ months old. ${ }^{6,11,13,17}$ Most published series of cervical fusion in the pediatric population include cohorts that are generally older than 3 years of age. ${ }^{10}$

We present a case of complete fracture dislocation of the subaxial cervical spine in a 3-week-old infant result-

ABBREVIATIONS ASIA = American Spinal Injury Association; BMP = bone morphogenetic protein; rhBMP = recombinant human BMP.

SUBMITTED November 15, 2014. ACCEPTED March 3, 2015.

INCLUDE WHEN CITING Published online November 27, 2015; DOI: 10.3171/2015.3.PEDS13568. 
ing from nonaccidental trauma. The neurological sequelae and medical and surgical management are discussed. To our knowledge, this represents the first report of a posterior cervical fusion in a child less than 1 month of age. Given the severity of the injury and the exceedingly young age of the patient, the specific technical and mechanical challenges of surgical stabilization of the spine are addressed.

\section{Case Report}

History

A 3-week-old, white, female infant with no significant birth or medical history was brought to a military hospital with a parental report of diminished movement and irritability. The patient's father reported that the child had fallen from his arms and struck her head on a changing table 2 days prior to presentation. Clinicians noted her to be lethargic and bruising was noted over the child's neck.

Multiple attempts at lumbar puncture were unsuccessful, and, therefore, broad-spectrum antibiotic therapy was initiated for meningitis coverage. Of note, the patient was also found to have hypothermia and hyponatremia, and appropriate treatment was initiated. A noncontrast CT study of the head was obtained and showed no evidence of any intracranial abnormality, and a chest radiograph demonstrated a right clavicle fracture.

The infant was subsequently transferred to a community hospital where a skeletal survey demonstrated a distraction injury between $\mathrm{C}-5$ and $\mathrm{C}-6$, possible healing bilateral seventh and eighth rib fractures, and the clavicle fracture. Given the multiple injuries and concern for nonaccidental trauma, the child was placed into the custody of the local Division of Family and Children Services (DFCS) and the father was taken into police custody. Subsequent further investigative action failed to reveal any definitive information regarding the mechanism of injury.

The infant was then transferred to our pediatric tertiary care hospital for definitive care. Strict cervical spine precautions were maintained throughout transport.

\section{Examination}

The patient was admitted directly to the pediatric intensive care unit. Upon presentation, she was intubated and ventilated. Her cranial sutures were approximated and her anterior fontanelle was open, soft, and flat. All 4 extremities were hypotonic with no spontaneous movement or movement to stimulation. She exhibited triple flexion in both lower extremities. Her initial examination was consistent with an American Spinal Injury Association (ASIA) classification of A, with no evidence of preserved motor or sensory function below the level of injury.

$\mathrm{CT}$ of the head and cervical spine was repeated upon admission. There remained no acute abnormality on head $\mathrm{CT}$, however spine imaging demonstrated a distraction injury with $8 \mathrm{~mm}$ of separation between the vertebral bodies of C-5 and C-6 (Fig. 1 A-C). MRI of the complete spine was performed, as were MRI, arteriography, and venography of the brain.

There was no visible anterograde flow in the left vertebral artery in the neck above the level of C-6; however, there was distal retrograde reconstitution from the contralateral side. A small focus consistent with embolic infarc-

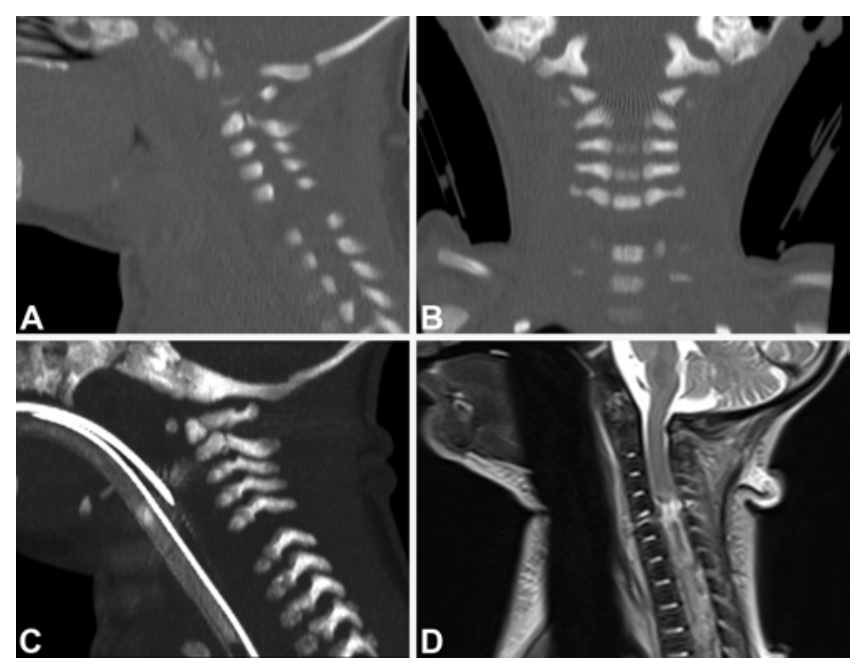

FIG. 1. A-C: Preoperative sagittal (A), coronal (B), and 3D maximum intensity projection (C) CT images demonstrating a severe distraction injury at C5-6. D: Sagittal T2-weighted MR image demonstrating a spinal cord injury at this level with near-complete transection.

tion was detected adjacent to the atrium of the left lateral ventricle. In the spine, there was complete or near-complete transection of the spinal cord at C5-6 with edema present from C-4 to T-4 (Fig. 1D). Hemorrhage within the epidural, subdural, and subarachnoid space was present throughout the cervical, thoracic, and lumbar spine.

At C5-6, there was complete disruption of the anterior and posterior longitudinal ligaments, ligamentum flavum, and interspinous ligament with preservation of the nuchal ligament. The facet joints were completely disrupted bilaterally and the intervertebral disc was avulsed from the superior endplate of C-6.

\section{Operation}

Elective surgical stabilization of the spine was performed to allow comprehensive and aggressive rehabilitation. The infant was taken to the operating room for an open reduction of the cervical dislocation, posterior segmental fusion using rib autograft, and augmentation with bone morphogenetic protein.

She was positioned prone on a horseshoe head holder. An initial incision was made over the ninth and tenth ribs on the patient's left side, and these rib segments were harvested to serve as structural autograft (Fig. 2). Subsequently, a midline posterior cervical incision was made from C-3 to T-1. While complete disruption of the C5-6 interspinous ligament was observed, localization was confirmed with fluoroscopy.

Sublaminar fixation was performed by passing a \#2 silk below the lamina bilaterally from C-5 to C-6. Next, umbilical tape was passed in a sublaminar fashion from $\mathrm{C}-4$ to C-7. The bilateral \#2 silk sutures were then carefully tied to reduce the distraction under fluoroscopic guidance, which demonstrated excellent reduction. The rib grafts were then split longitudinally and secured in the posterolateral gutters using the umbilical tape. The remaining ribs were then wrapped in 4.2-mg recombinant human bone morphogenetic protein (rhBMP-2) patties and were placed from C-4 to C-7. 


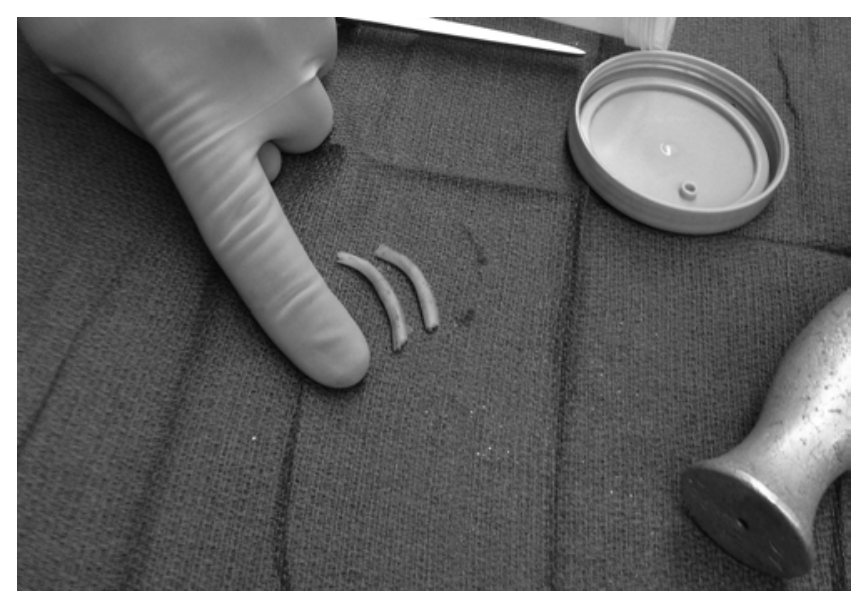

FIG. 2. Intraoperative photograph showing the rib autograft that was harvested and used for posterolateral stabilization and fusion.

\section{Postoperative Course}

Postoperatively, cervical immobilization was continued with a rigid cervical collar. Not unexpectedly, the patient could not be weaned from ventilator support and therefore underwent a tracheostomy. There was some difficulty in obtaining a collar appropriately sized for a 1-month-old infant that could accommodate the tracheostomy within the cervical collar. A commercially available cervical orthotic was modified to create an aperture in the front plate of the brace. A percutaneous endoscopic gastrostomy tube was also placed for enteral feeding.

Two months following surgery, CT imaging of the cervical spine was performed to evaluate the cervical alignment, as well as the quantity and quality of bony fusion. There was reduction of the prior distraction between C-5 and C-6 with only minimal residual widening (Fig. 3). At this point, significant bilateral bridging bone was demonstrated, consistent with a fusion grade of 3 based on a previously published CT fusion scale. ${ }^{7}$ A small posterior rim of bone developed within the canal at the level of injury. However, this measured less than $1.3 \mathrm{~mm}$ in its greatest anteroposterior diameter and did not cause any significant stenosis. Given significant bony fusion, the cervical collar was removed at this time.

Approximately 3 months after surgery, the patient was transferred out of state to another hospital for continued inpatient rehabilitation with the custodial parent. At the time of transfer, she had good upper-extremity movement but poor distal upper-extremity strength. With the exception of hip flexion, her lower-extremity strength was weaker than antigravity. Medical and rehabilitation records as well as imaging studies were obtained to allow for additional follow-up. Follow-up MRI demonstrated chronic myelomalacia at the level of injury, as well as posttraumatic pseudomeningoceles at C5-6 likely resulting from the avulsion of the bilateral C-6 nerve roots (Fig. 4). At 2 years old, the patient remained dependent on a gastrostomy tube and tracheostomy. She is continuing to be weaned from the ventilator and is currently spending several hours each day off ventilatory support. She continues to participate in physical and occupational therapy with good progression. She has demonstrated improvement in upper-extremity
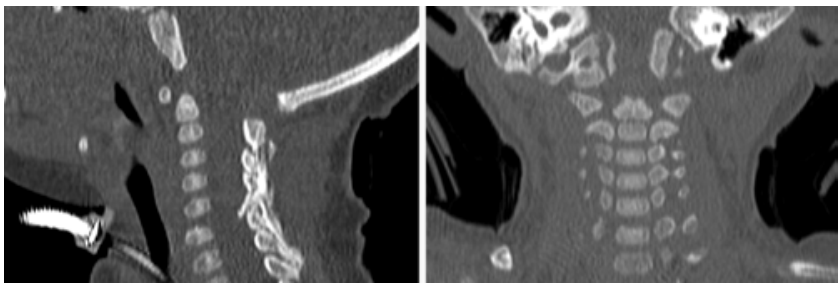

FIG. 3. Postoperative CT reconstructions in the sagittal (left) and coronal (right) planes showing restoration of cervical alignment and evolving posterolateral fusion mass.

movement, with improved strength and dexterity in both upper extremities. Her ultimate neurological examination findings were consistent with an ASIA C classification at the C-6 level.

\section{Discussion}

Spinal fusion procedures in neonatal patients pose specific technical and mechanical challenges related to the distinct anatomical differences found in this population. Namely the large relative head size, immaturity of the axial neck musculature, laxity of spinal ligaments, and incomplete bone ossification. It is these same anatomical characteristics that predispose these patients to more severe spinal cord injuries. Further, the use of instrumentation in these patients is not feasible. In our patient, difficulty also existed in the identification of an appropriate rigid cervical collar. Available neonatal cervical orthotics did not contain an aperture to accommodate a tracheostomy, and therefore customization of the front plate was required with care taken to minimize the removal of material so as to not compromise its structural integrity. As the patient grew, commercially available orthotics were available with anterior tracheostomy apertures.

The risk of cervical spine injury in cases of nonaccidental trauma is high, but these injuries most frequently occur at the cervicomedullary junction and less often in the subaxial cervical spine. ${ }^{4}$ Rooks et al. presented a report of

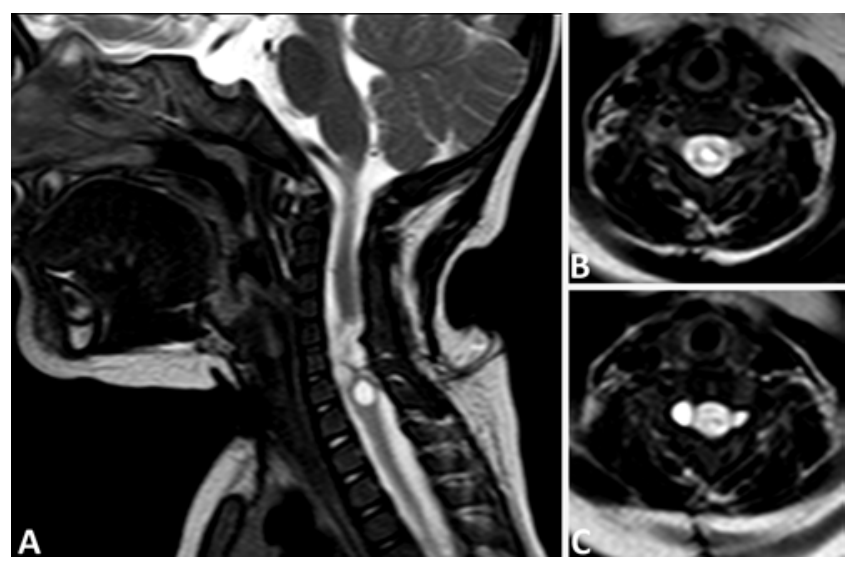

FIG. 4. Postoperative MR images obtained 16 months after surgery. T2-weighted sagittal image (A) and axial images obtained at C-5 (B) and C5-6 (C) demonstrating the severe spinal cord injury with nearcomplete transection. Note the bilateral meningeal diverticula present in Panel $\mathrm{C}$, which are likely indicative of traumatic avulsion of the C- 6 nerve roots. 
cervical spine injury in twin 3-month-old infant siblings. ${ }^{15}$ In their report, the first infant had suffered a compression fracture at C-5 and anterolisthesis of C-4 on C-5 with cord compression that required decompressive laminectomy from C4-7 and C3-7 posterior fusion. However, surgical details were not reported. The second child, also 3 months of age at the time of injury, suffered a fracture-dislocation at C-5 on C-6 with mild cord compromise. Initial treatment in this child was with external immobilization, but progressive deformity and instability warranted surgical intervention, and 3 surgical procedures, beginning at age 7 months, were required for spinal stabilization. ${ }^{15}$

While there are few studies regarding the use of BMP in a pediatric population, it has been demonstrated to be safe and efficacious in posterior spinal fusion procedures. ${ }^{5} \mathrm{In}$ infants, there is a single report of salvage use of rhBMP-2 in a 16-month-old child with Down syndrome for failed occipitocervical fusion after 2 prior attempts. During the third operation, rhBMP-2 was used successfully with subsequent solid bony fusion. ${ }^{11}$ In another report, Benzel et al. described the use of rhBMP to augment occipitocervical fusion in a 12-month-old child who had sustained an atlantooccipital dislocation. ${ }^{1}$

We believe that our report is the first reported use of rhBMP-2 in a child under the age of 1 year. There are several justifications to support the use rhBMP in this case. The injury sustained by the infant was purely ligamentous, and such injuries are historically associated with lower fusion rates. In addition, the cervical vertebrae in the neonate are so thin that decortication is not feasible and can easily jeopardize the structural integrity of the bone. As demonstrated in our case, rigid immobilization is challenging in this age group and can also impair fusion. There is no precedent for cervical fusion in neonates, and the literature includes no studies of fusion rates in this population. The most significant potential risk of rhBMP use in this case was felt to be that of heterotopic ossification. While not clinically significant, the ectopic development of a small amount of bone posteriorly within the spinal canal in our patient may be related to the use of rhBMP-2.

Our case is, to our knowledge, the first case of posterior cervical spinal fusion in a 3-week-old infant for a C5-6 complete fracture dislocation that occurred as the result of nonaccidental trauma. Despite the challenges of operative fusion in the infant, we were nonetheless able to achieve reduction of the dislocation, restoration of normal cervical alignment, and solid bony fusion.

\section{References}

1. Benzel EC, Zhang DH, Iannotti C, Refai D, Ruggieri P, Krishnaney A: Occipitocervical fusion in an infant with atlantooccipital dislocation. World Neurosurg 78:715.e717715.e24, 2012

2. Bresnan MJ, Abroms IF: Neonatal spinal cord transection secondary to intrauterine hyperextension of the neck in breech presentation. J Pediatr 84:734-737, 1974

3. DeVivo MJ, Vogel LC: Epidemiology of spinal cord injury in children and adolescents. J Spinal Cord Med 27 (Suppl 1):S4-S10, 2004

4. Eleraky MA, Theodore N, Adams M, Rekate HL, Sonntag VKH: Pediatric cervical spine injuries: report of 102 cases and review of the literature. J Neurosurg 92 (1 Suppl): $12-17,2000$

5. Fahim DK, Whitehead WE, Curry DJ, Dauser RC, Luerssen TG, Jea A: Routine use of recombinant human bone morphogenetic protein-2 in posterior fusions of the pediatric spine: safety profile and efficacy in the early postoperative period. Neurosurgery 67:1195-1204, 2010

6. Gaufin LM, Goodman SJ: Cervical spine injuries in infants. Problems in management. J Neurosurg 42:179-184, 1975

7. Glassman SD, Dimar JR, Carreon LY, Campbell MJ, Puno RM, Johnson JR: Initial fusion rates with recombinant human bone morphogenetic protein-2/compression resistant matrix and a hydroxyapatite and tricalcium phosphate/collagen carrier in posterolateral spinal fusion. Spine (Phila Pa 1976) 30:1694-1698, 2005

8. Kewalramani LS, Tori JA: Spinal cord trauma in children. Neurologic patterns, radiologic features, and pathomechanics of injury. Spine (Phila Pa 1976) 5:11-18, 1980

9. Kokoska ER, Keller MS, Rallo MC, Weber TR: Characteristics of pediatric cervical spine injuries. J Pediatr Surg 36:100-105, 2001

10. Lowry DW, Pollack IF, Clyde B, Albright AL, Adelson PD: Upper cervical spine fusion in the pediatric population. $\mathbf{J}$ Neurosurg 87:671-676, 1997

11. Lu DC, Sun PP: Bone morphogenetic protein for salvage fusion in an infant with Down syndrome and craniovertebral instability. Case report. J Neurosurg 106 (6 Suppl): 480-483, 2007

12. Mohseni S, Talving P, Branco BC, Chan LS, Lustenberger $\mathrm{T}$, Inaba K, et al: Effect of age on cervical spine injury in pediatric population: a National Trauma Data Bank review. J Pediatr Surg 46:1771-1776, 2011

13. Mubarak SJ, Camp JF, Vuletich W, Wenger DR, Garfin SR: Halo application in the infant. J Pediatr Orthop 9:612-614, 1989

14. Platzer P, Jaindl M, Thalhammer G, Dittrich S, KutschaLissberg F, Vecsei V, et al: Cervical spine injuries in pediatric patients. J Trauma 62:389-396, 2007

15. Rooks VJ, Sisler C, Burton B: Cervical spine injury in child abuse: report of two cases. Pediatr Radiol 28:193-195, 1998

16. Schottler J, Vogel LC, Sturm P: Spinal cord injuries in young children: a review of children injured at 5 years of age and younger. Dev Med Child Neurol 54:1138-1143, 2012

17. Weiss MH, Kaufman B: Hangman's fracture in an infant. Am J Dis Child 126:268-269, 1973

\section{Disclosures}

The authors report no conflict of interest concerning the materials or methods used in this study or the findings specified in this paper.

\section{Author Contributions}

Conception and design: all authors. Acquisition of data: all authors. Analysis and interpretation of data: all authors. Drafting the article: all authors. Critically revising the article: all authors. Reviewed submitted version of manuscript: all authors.

\section{Supplemental Information}

\section{Previous Presentations}

Portions of this work were previously presented at the spring meeting of the Georgia Neurosurgical Society in Sea Island, Georgia, May 23-25, 2014.

\section{Correspondence}

David M. Wrubel, Pediatric Neurosurgical Associates, Children's Healthcare of Atlanta, 5455 Meridian Mark Rd. NE, Ste. 540, Atlanta, GA 30342-1654. email: dwrubel@emory.edu. 